\title{
EMPLEO DE ESPECTROSCOPIA RAMAN, DIFRACCIÓN DE RAYOS X Y MICROSCOPIA ELECTRÓNICA PARA EL ANÁLISIS DE PIGMENTOS EN CERÁMICAS VAQUERÍAS
}

\section{THE USE OF RAMAN SPECTROSCOPY, X-RAY DIFFRACTION AND ELECTRON MICROSCOPY IN THE ANALYSIS OF PIGMENTS FROM VAQUERIAS CERAMICS}

\author{
M. Fabiana Bugliani*, Claudia DI \\ LELLO** ELEONORA FreIRE *** GrISELDA \\ POlla $* * * *$, AllCIA Petragalli ${ }^{\prime} * \star \star \star *$, \\ MARÍA REINOSO****** \& EMILIA B. \\ $\mathrm{HALAC}^{* \star * * * * \star}$
}

Se presentan resultados del estudio arqueométrico de pigmentos rojos y negros provenientes de diseños pintados en cerámicas Vaquerías, estilo polícromo del Período Formativo del Noroeste Argentino. Se combinan las técnicas de espectroscopia Raman, difracción de rayos X (DRX) y microscopia electrónica de barrido con espectroscopia de energía dispersiva (SEM-EDS) para analizar la composición de los pigmentos en fragmentos de piezas cerámicas de los sitios Cardonal y Bordo Marcial (Catamarca, Argentina). Los pigmentos rojos están compuestos esencialmente de hematita; en los pigmentos negros se determinó alto contenido de manganeso y se confirmó la presencia de jacobsita y bixbyita.

Palabras clave: Vaquerías, Noroeste Argentino, DRX, Raman, SEM-EDS, manganeso

This paperpresents the results of using archaeometric techniques to analyze red and black pigments from designs painted onto Vaquerias ceramics (a polychrome style of the Argentine Northwest Formative period). Raman spectroscopy, X-ray diffraction (XRD) and scanning electron microscopy with energy dispersive spectroscopy (SEM-EDS) were all employed in the compositional analysis of pigments on ceramic pieces from Cardonal and Bordo Marcial (Catamarca, Argentina) that were inhabited during the first two centuries of the Christian era. The red pigments have proven to be primarily hematite, while black pigments showed high contents of manganese and the presence of jacobsite and bixbyite.

Key words: Vaquerias, Northwestern Argentina, XRD, Raman, SEM-EDS, manganese

* M. Fabiana Bugliani, Conicet-Museo Etnográfico J. B. Ambrosetti, Universidad de Buenos Aires, Argentina, email: fbugliani@gmail.com * Claudia Di Lello, CICpBA-Museo de La Plata-FCNYM-UnLP, email: cdilello@fenym.unlp.edu.a

** Eleonora Freire, CNEA, Buenos Aires, Argentina, email: freire@tandar.cnea.gov.ar

*****aselda Polla, CNEA, Buenos Aires, Argentina, email: grispoll@cnea.gov.ar

****** Alicia Petragalli, CNEA, Buenos Aires, Argentina, email: petra@tandar.cnea.gov.ar

*******: María Reinoso, CNEA, Buenos Aires, Argentina, email: reinoso@cnea.gov.ar

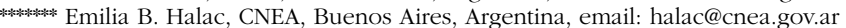

Recibido: marzo de 2012. Aceptado: febrero de 2013.

\section{INTRODUCCIÓN}

La alfarería polícroma más antigua del Noroeste Argentino (NOA) se conoce como Vaquerías (Heredia et al. 1974). Es un estilo pintado, de elaborada terminación de superficie, en el cual predominan los diseños geométricos.

El repertorio de formas conocido para Vaquerías incluye algunas siluetas características y otras menos frecuentes. Entre las formas típicas se observan los vasos cilíndricos y subcilíndricos de paredes levemente cóncavas, con bordes a veces evertidos, labio recto o convexo, base plana y asa vertical. También se incluyen jarras de cuerpo subglobular con cuello corto cilíndrico o de paredes levemente cóncavas, bordes evertidos, muchas veces engrosados y con una línea excisa en la unión cuello-cuerpo que resalta el punto angular (fig. 1). De igual manera, existen cuencos de perfil compuesto, con punto angular en el tercio inferior de la altura de la pieza, paredes suavemente cóncavas y borde engrosado.

Además se han detectado otras formas menos representadas, como cuencos hemisféricos de perfil simple y vasijas ovoides de cuello corto. Existen algunos ejemplos de piezas escultóricas en las que, además de utilizarse 

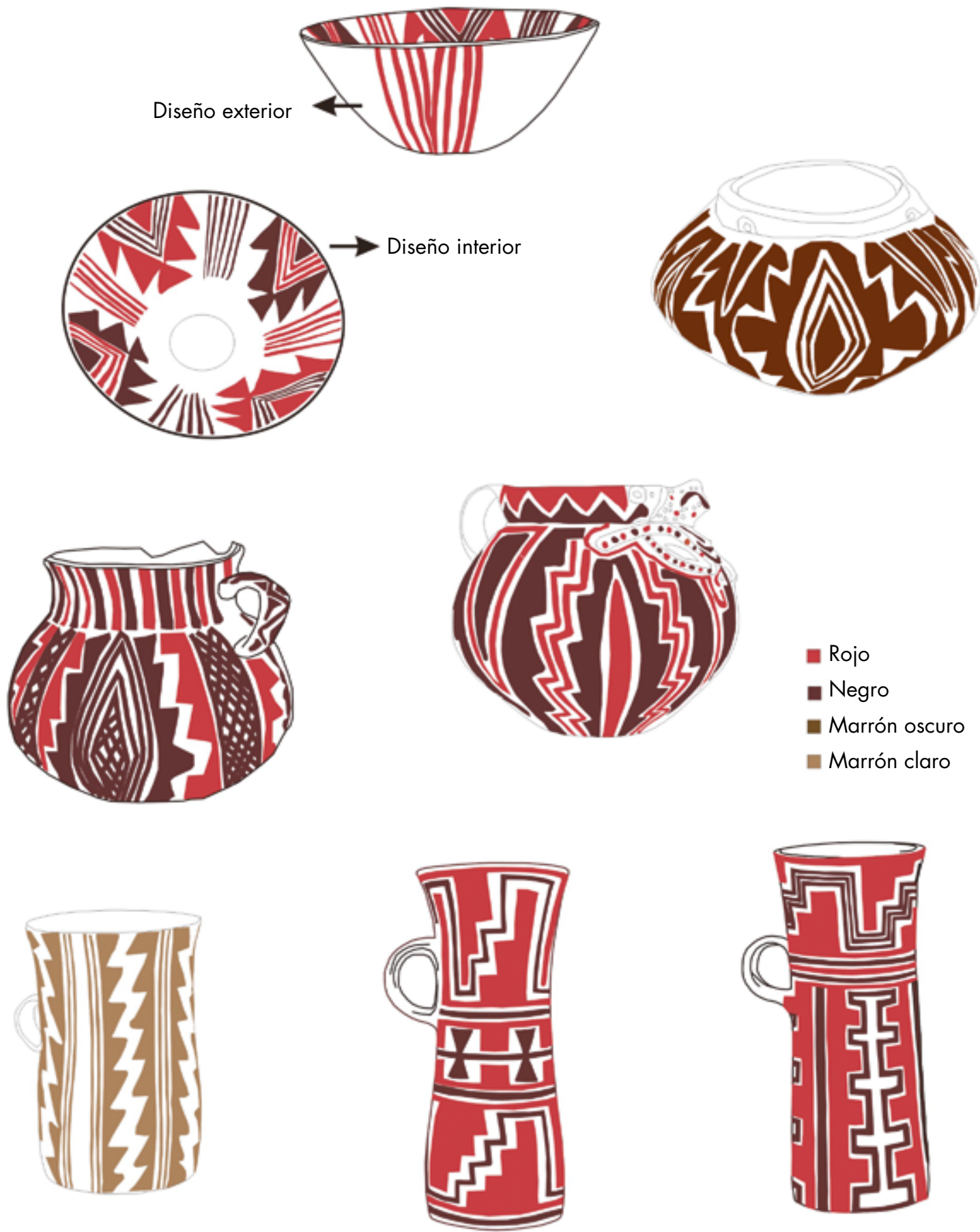

Figura 1. Repertorio de formas de vasijas de estilo Vaquerías. Figure 1. Assorted Vaquerías vessel forms.

la pintura con motivos geométricos, se han modelado figuras humanas y animales.

Las representaciones visuales de este estilo están construidas a partir de figuras geométricas sólidas (cuadrados, rombos, triángulos en hilera), de líneas (paralelas, escalonadas, rombos concéntricos) y reticulados que se combinan según reglas de simetría. La configuración final es resultado del uso de estas reglas junto con una precisa selección de colores y tonalidades. En este estilo destaca la pintura en negro y rojo en distintos tonos. 
Las representaciones se disponen sobre el fondo claro anaranjado propio de las pastas Vaquerías y, en algunas oportunidades, sobre un engobe blanco amarillento que fue aplicado como fondo.

Por medio de análisis petrográfico de pastas en piezas Vaquerías halladas en el valle del Cajón (Catamarca, Argentina) se ha reconocido el uso de arcillas a las cuales se les agregaron inclusiones de pizarra-filita, cuarzo, arenisca y tiesto molido. En estas pastas se observan escasas cavidades y las inclusiones se encuentran bien cementadas en la matriz arcillosa. Esto permite pensar en una tradición artesanal que implicó un buen trabajo de amasado y la búsqueda de altas temperaturas con el fin de lograr pastas compactas y de alta dureza, que no se desgranan a pesar del alto porcentaje de inclusiones no plásticas (Pereyra Domingorena 2010). Dado el uso de litoclastos metamórficos (pizarra-filita) como atemperantes, los cuales no están presentes en la geología local, se estableció el carácter alóctono de estas vasijas en el valle del Cajón (Pereyra Domingorena 2010; Bugliani \& Pereyra Domingorena 2012).

La cerámica Vaquerías pertenece al conjunto de bienes que ha participado en las redes de interacción que se establecieron por todo el Noroeste Argentino y áreas vecinas durante el Período Formativo (González \& Baldini 1989; Tartusi \& Núñez Regueiro 1993; Korstanje 1995; Lazzari 2005). Esto es congruente con la extendida presencia de este estilo en diferentes ambientes del NOA e incluso en el norte de Chile. La amplia dispersión de Vaquerías incluye su hallazgo en los valles de Hualfín, Santa María, Calchaquí Norte, del Cajón, Tafí, Lerma, Quebrada del Toro, Puna y Yungas. También se han encontrado fragmentos de este estilo en algunos sitios de la subregión del río Salado y de San Pedro de Atacama en Chile (para una revisión reciente de los sitios con cerámica Vaquerías ver Bugliani y Pereyra Domingorena 2012).

Cronológicamente esta alfarería ha sido datada entre el 200 AC y 400 DC (Heredia et al. 1974). Esta primera asignación posteriormente fue reafirmada a partir de nuevos hallazgos en diferentes sitios del NOA y, particularmente en Quebrada del Toro (Salta), existen fechados que amplían la presencia de Vaquerías desde aproximadamente el 300 AC hasta finales del primer milenio de la era cristiana (De Feo 2010).

Dado que el uso de la policromía es uno de los aspectos más destacados de esta cerámica temprana, consideramos relevante indagar los aspectos tecnológicos de la ejecución de los diseños pintados, buscando identificar las materias primas utilizadas para pintar las piezas. El objetivo de este trabajo es la identificación y caracterización de los pigmentos utilizados para pintar estas vasijas a partir de la aplicación de técnicas fisicoquímicas.

\section{MATERIALES Y MÉTODOS}

\section{Las muestras}

Las muestras analizadas proceden de los sitios formativos Cardonal y Bordo Marcial, situados en el valle del Cajón (Departamento de Santa María, Catamarca, Argentina) y distantes entre sí dos kilómetros aproximadamente (fig. 2). Estos contextos domésticos aldeanos donde aparece cerámica Vaquerías asociada a otros estilos locales presentan fechados que se ubican en los dos primeros siglos DC (Scattolin et al. 2009a y b).

En estos sitios, la cerámica de pastas finas representa alrededor del $20 \%$ del total de fragmentos recuperados y el porcentaje de tiestos decorados es aún menor. En las excavaciones sistemáticas efectuadas en Cardonal y Bordo Marcial se han obtenido 35 fragmentos Vaquerías correspondientes a distintas piezas (fig. 3), de los cuales seis fueron seleccionados para hacer la caracterización por distintas técnicas fisicoquímicas. Se eligieron fragmentos pintados en diferentes tonalidades de rojos y negros sobre el engobe blanco amarillento, de manera de cubrir distintas variantes de la policromía.

\section{Técnicas utilizadas}

Las muestras estudiadas (fig. 4) fueron analizadas mediante las técnicas de difracción de rayos X (DRX), microscopia electrónica de barrido con espectroscopia de energía dispersiva (SEM-EDS) y espectroscopia Raman (RS), en los laboratorios del Centro Atómico Constituyentes, Comisión Nacional de Energía Atómica (CNEA).

La caracterización morfológica y la composición elemental fueron analizadas por SEM-EDS empleando un equipo Philips 515 microscope (Philips Export B. V., Eindhoven, The 134 Netherlands) equipado con sonda EDS EDX PV9100 probe (EDX International Inc., Prairie, View, 135 IL, USA). Todos los espectros fueron obtenidos a $20 \mathrm{kV}$ y con un tiempo de análisis de $40 \mathrm{~s}$. Dado que los fragmentos fueron analizados sin tratamiento previo, esta técnica no es destructiva. Los tiestos se montaron en un portamuestras de aluminio con cinta de grafito (eléctricamente conductor). Se observaron y fotografiaron a distintos aumentos y se hizo el análisis elemental sobre diversas partes de las muestras. Este método de análisis permite detectar la presencia de elementos cuyo peso atómico sea mayor que 5. 


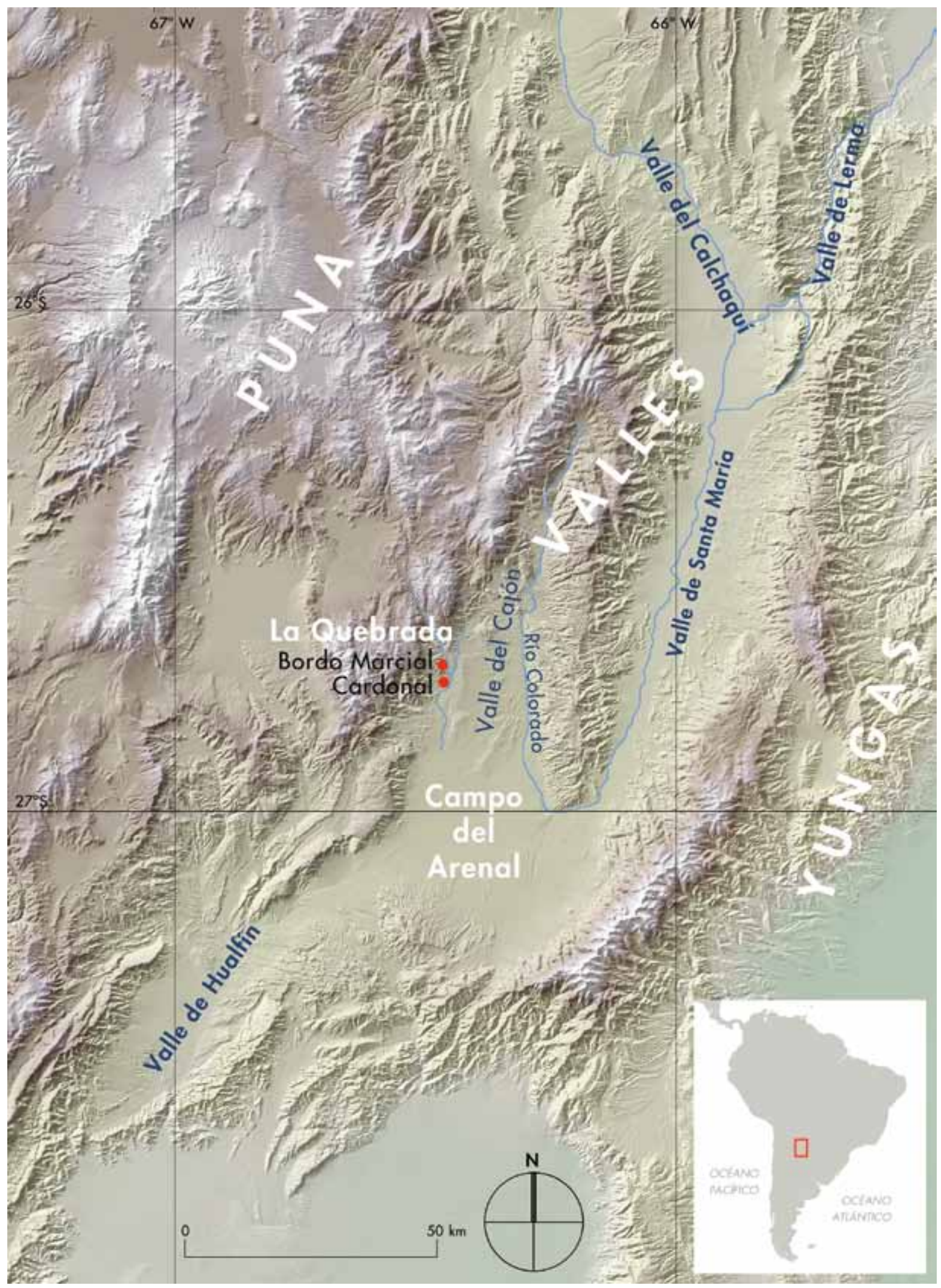

Figura 2. Área de estudio con la localización de Cardonal y Bordo Marcial, sitios de donde proceden las muestras Vaquerías analizadas. Figure 2. Area of study indicating the locations of Cardonal and Bordo Marcial, where the Vaquerias pieces were found. 


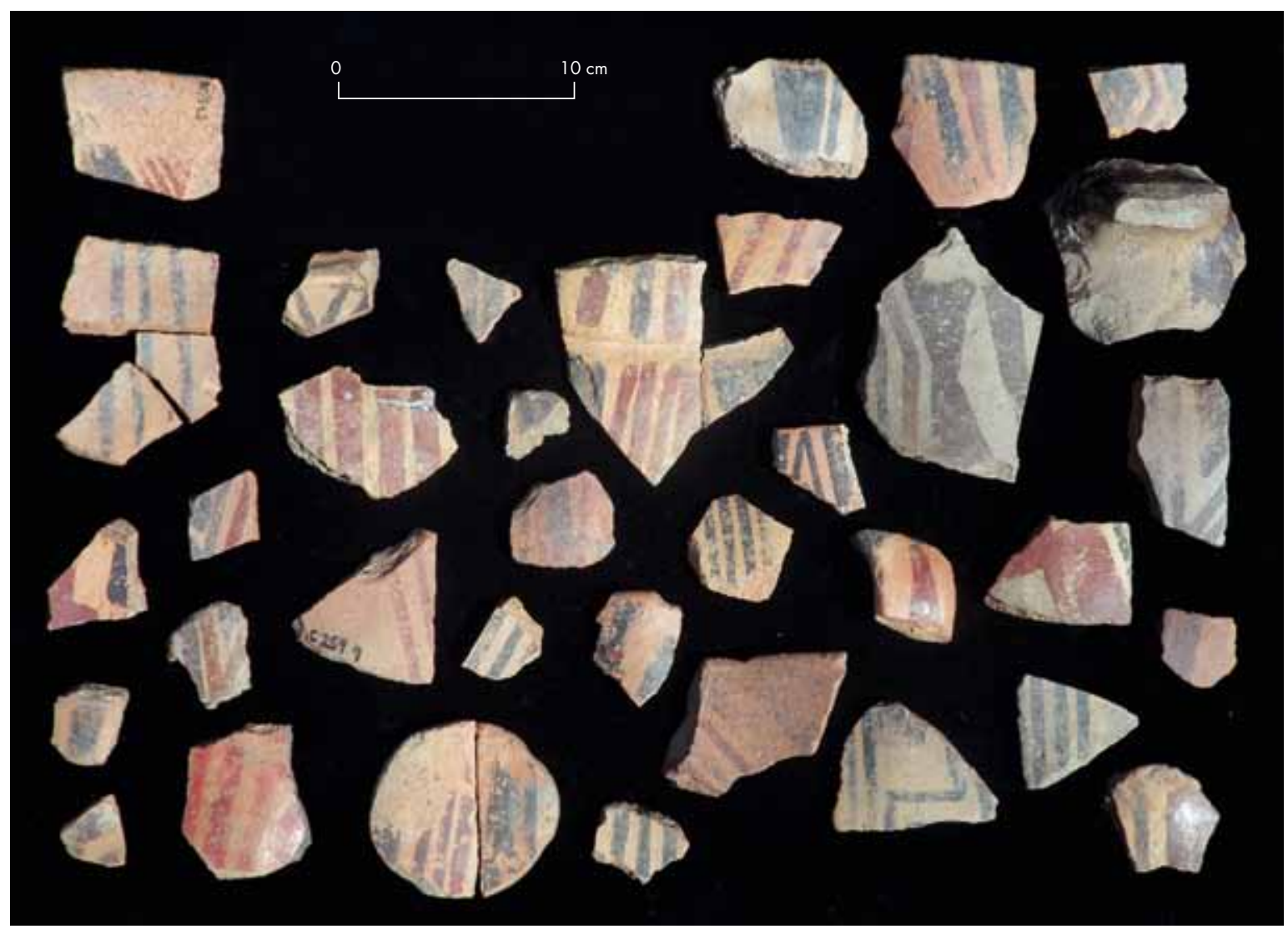

Figura 3. Muestra de fragmentos Vaquerías recuperados en Cardonal y Bordo Marcial.

Figure 3. Vaquerias sherds recovered from Cardonal and Bordo Marcial.

La técnica de difracción de rayos X (DRX) fue utilizada para la identificación de las fases cristalinas presentes en las muestras. La adquisición de los datos se realizó en el rango de $10^{\circ}<2 \theta<70^{\circ}$ empleando un equipo Philips PW 3710 con monocromador y radiación de $\mathrm{Cu}(\mathrm{K}-\mathrm{Alph} 1[\AA]=1.54060)$. Se utilizó un paso de $0.02^{\circ}$ en $2 \theta$ con un tiempo de contaje de dos segundos por paso y con un generador de $30 \mathrm{~mA}, 40 \mathrm{kV}$. Para la identificación de fases presentes se empleó un programa denominado PC-Identify y la base de datos de la ICDD. Los fragmentos cerámicos fueron analizados sin tratamiento ni preparación previa. Si bien la técnica de difracción de rayos $\mathrm{X}$ es preferentemente usada para muestras molidas a polvo, utilizar los fragmentos cerámicos permite ganar información sobre la composición superficial en relación con la matriz y, en particular, sobre los pigmentos utilizados para realizar los diseños. El haz de rayos X se direccionó sobre la zona decorada o con pigmentos a estudiar.

Los espectros Raman fueron adquiridos en un espectrómetro comercial LabRAM HR (Horiba Jobin Yvon) equipado con doble monocromador, una red de difracción de 1800 líneas/mm y detector CCD (Charge Coupled Device) de 1024 x 256 pixeles, con resolución espectral de $1.2 \mathrm{~cm}^{-1}$. Se utilizó como fuente excitadora la línea $514.5 \mathrm{~nm}$ de un láser de argón. La potencia del láser fue controlada $(<0.2 \mathrm{~mW}$ sobre la muestra) con el fin de evitar el calentamiento y consecuente degradación de la muestra. Se utilizaron objetivos de x10 y x50 en el microscopio, concentrando el haz en un área circular de $\sim 10$ y $5 \mu \mathrm{m}$ de diámetro, respectivamente. Se analizaron del orden de diez regiones en cada muestra, barriendo áreas aproximadamente circulares de $100 \mu \mathrm{m}$ de diámetro, en cada caso.

\section{RESULTADOS}

La caracterización por SEM y EDS se realizó sobre los seis fragmentos seleccionados analizando la morfología y la composición en varios puntos, tanto en la zona correspondiente a la base como sobre las zonas decoradas con pigmentos rojos y negros. La mayoría de las muestras contienen $\mathrm{Al}, \mathrm{C}, \mathrm{Ca}, \mathrm{K}, \mathrm{O}, \mathrm{Si}$ y Ti, cuya 

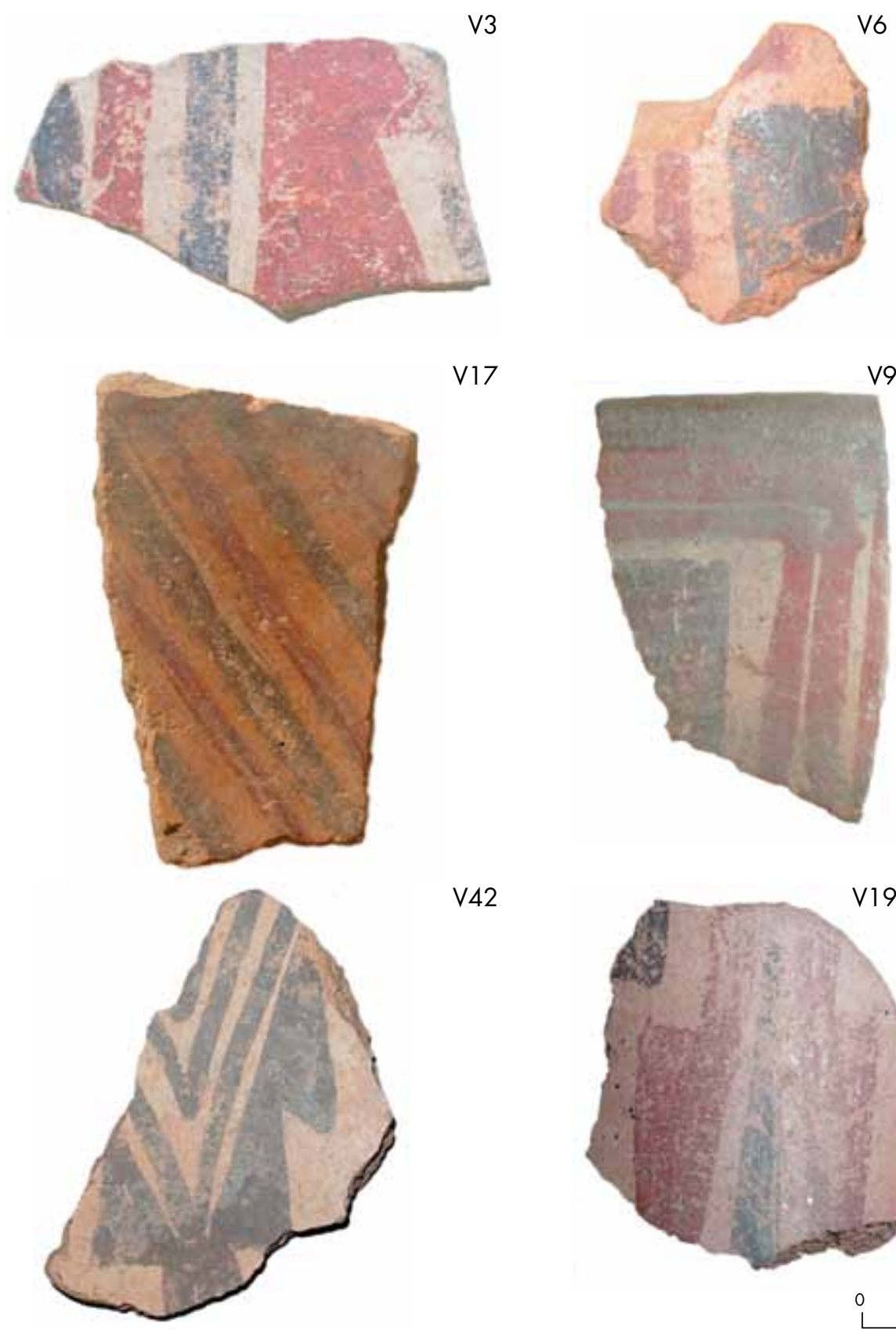

V17

V9
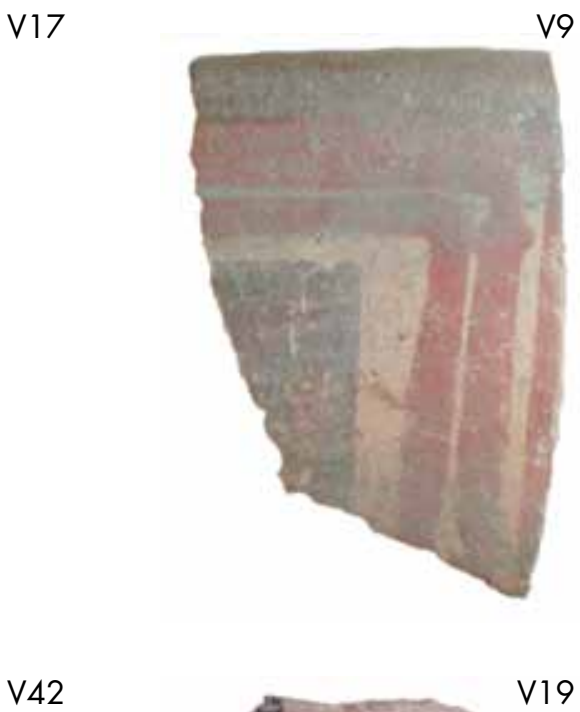

V42

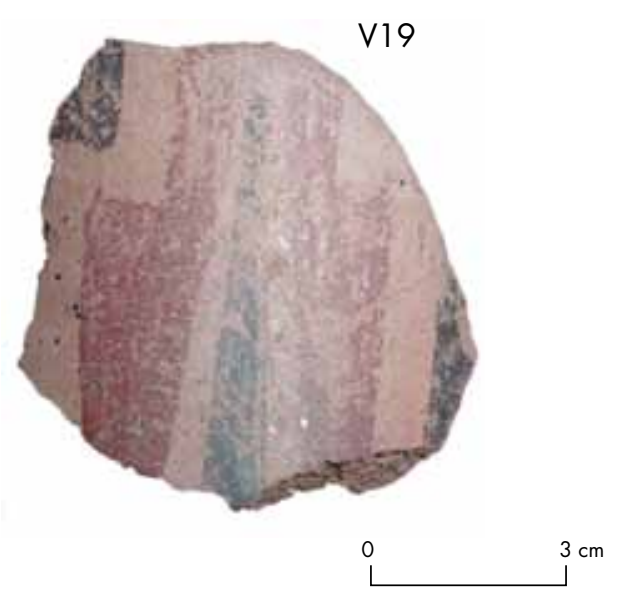

Figura 4. Los fragmentos estudiados.

Figure 4. Sherds analyzed.

concentración varía de acuerdo con la fase cristalina de la pasta, además de la presencia de otros elementos que son característicos de cada pigmento. En las zonas rojas, el componente dominante es hierro (Fe), mientras que en las zonas negras es mayoritario el contenido de Fe y manganeso ( $\mathrm{Mn}$ ) en similares proporciones. El porcentaje de $\mathrm{C}$ no es significativo y por lo tanto se descarta que el carbón sea un componente utilizado en los pigmentos negros. A modo de ejemplo se presentan los resultados de SEM-EDS para la muestra V19 en la zona roja (fig. 5a) y negra (fig. 5b).

$\mathrm{El}$ análisis por difracción de rayos $\mathrm{X}$ realizado a los tiestos cerámicos de Vaquerías dio como resultado la identificación de varios tipos de aluminosilicatos, cuarzo $\left(\mathrm{SiO}_{2}\right)$ y dióxido de titanio $\left(\mathrm{TiO}_{2}\right)$ en las fases de anatasa o rutilo, atribuidos a la pasta de la base. 

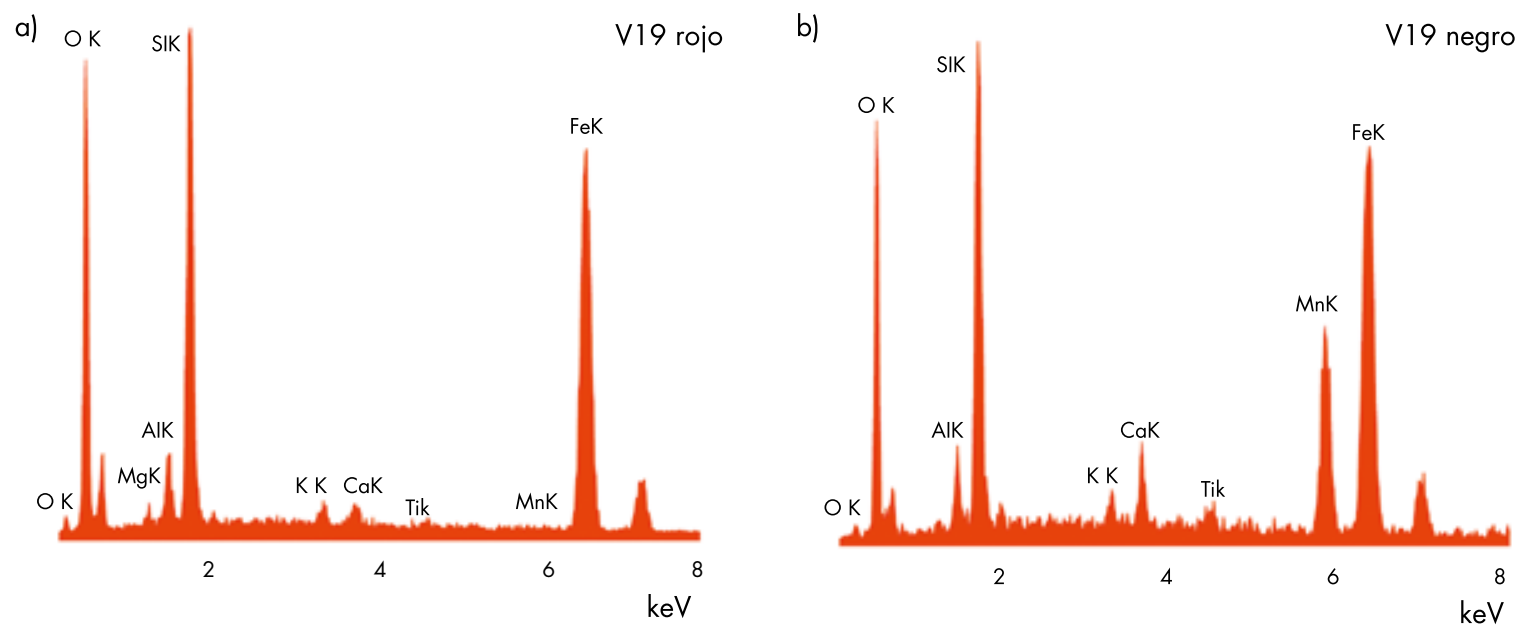

Figura 5. Composición elemental obtenida por SEM-EDS de dos puntos de la muestra V19: a) zona roja; b) zona negra. Figure 5. Elemental composition obtained from SEM-EDS from sample V19: a) red area; b) black area.

En el diagrama comparativo de DRX (fig. 6) podemos observar que las muestras V6, V9 y V19 tienen alto contenido de hematita $\left(\mathrm{Fe}_{2} \mathrm{O}_{3}\right)$. En ellas se observa bajo contenido de compuestos de Mn. La muestra V3, con predominio de diseño en rojo, tiene alto contenido de hematita, pero también una importante proporción de magnetita $\left(\mathrm{Fe}_{3} \mathrm{O}_{4}\right)$, bixbyita $\left[(\mathrm{Mn}, \mathrm{Fe})_{2} \mathrm{O}_{3}\right]$ y jacobsita $\left(\mathrm{MnFe}_{2} \mathrm{O}_{4}\right)$. La muestra $\mathrm{V} 42$, que solamente presenta diseños negros, revela alto contenido de magnetita aunque se detectan también jacobsita y hematita. En la muestra V17, con diseños de líneas paralelas negras y rojas, se observa hematita y un alto contenido de magnetita y bixbyita.

La técnica de espectroscopia Raman con enfoque microscópico permite analizar regiones pequeñas y por ello es posible diferenciar las zonas pintadas (en rojo o en negro) en una misma muestra (fig. 7). En los análisis realizados sobre el engobe destaca la presencia de cuarzo y anatasa, además de hematita y aluminosilicatos.

En todos los diseños rojos analizados (V3, V6, V9, V17 y V19) se confirma la presencia de hematita como componente mayoritario, en ocasiones acompañada de magnetita (fig. 7).

En relación con los diseños negros, en todas las muestras Vaquerías analizadas se observa la presencia de compuestos de Fe y de Mn, en la forma de magnetita y jacobsita principalmente (fig. 8). En algunos casos, se han detectado en regiones negras componentes de la base (hematita, cuarzo, aluminosilicatos), lo que ha sido atribuido a la porosidad de la capa pigmentaria. En ninguna de las muestras en estudio se detecta presencia de carbono como responsable del color negro.
En resumen, el componente dominante de los pigmentos rojos mostró ser óxido de hierro en forma de hematita $\left(\mathrm{Fe}_{2} \mathrm{O}_{3}\right)$. Del análisis de los pigmentos negros resalta la detección de compuestos de manganeso (bixbyita y jacobsita) junto con magnetita $\left(\mathrm{Fe}_{3} \mathrm{O}_{4}\right)$.

En investigaciones previas sobre material cerámico del NOA, se han asociado los pigmentos negros a compuestos de hierro (magnetita) y carbón (fundamentalmente en superficies internas) en vasijas de los períodos PDR e Inka de la Quebrada de Humahuaca (Botto et al. 1998). Por otra parte, también se ha reportado la presencia de compuestos de manganeso asociados a pigmentos negros; por ejemplo, $\mathrm{CaMnO}_{3}$ en cerámicas Portezuelo de ca. 500-1000 DC (Cremonte et al. 2003); pirolusita $\left(\mathrm{MnO}_{2}\right)$ y psilomelano $\left.\left[(\mathrm{Ba}, \mathrm{Mn})_{3}(\mathrm{O}, \mathrm{OH})_{6} \mathrm{Mn}_{8} \mathrm{O}_{16}\right)\right]$ observadas en tiestos Aguada Portezuelo de ca. 600-900 DC (De la Fuente \& Pérez Martínez 2008). Sin embargo, solo recientemente ha sido reportada la presencia de jacobsita en el NOA, en muestras de cerámicas Inka procedentes del valle Calchaquí-Yocavil de ca. 900-1530 DC (Centeno et al. 2012). En las muestras que nos ocupan no se detectaron reflexiones características de pirolusita (PDF\#00-024-0735), psilomelano (PDF\#00-006-0606) ni $\mathrm{CaMnO}_{3}$ (PDF\#00-003-0830), ni las bandas correspondientes en espectroscopia Raman. Sin embargo, se determinó la presencia de bixbyita y jacobsita.

\section{CONCLUSIONES}

La complementariedad en el uso de las técnicas de DRX, Raman y SEM-EDS ha demostrado ser útil para el análisis 


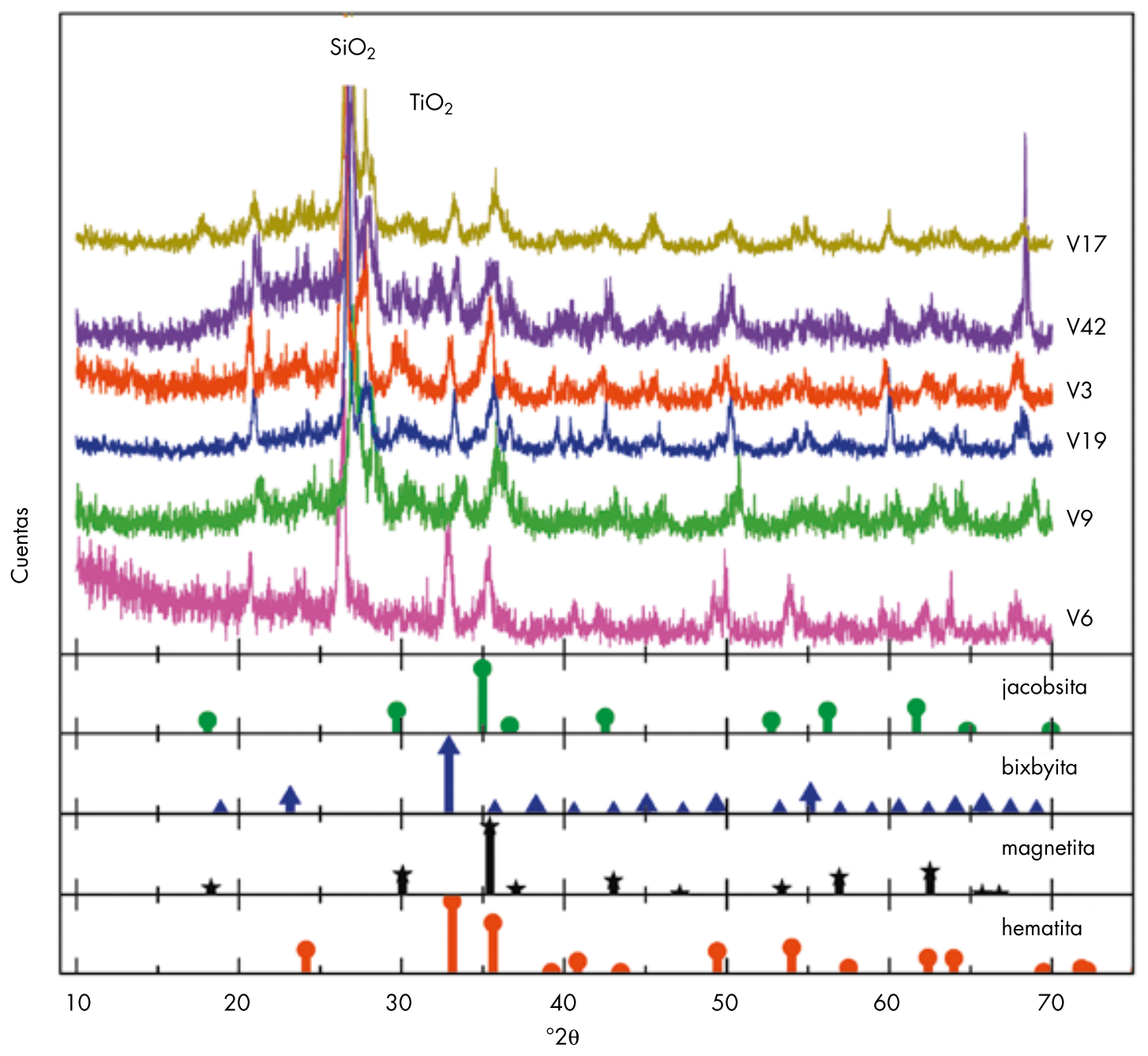

Figura 6. Difractogramas de rayos $\mathrm{X}$ de las muestras analizadas y de los patrones de jacobsita (PDF\#00-010-0319), bixbyita (PDF\#00-0100069), magnetita (PDF\#01-076-1849) y hematita (PDF\#00-024-0072).

Figure 6. X-ray diffraction patterns from samples studied and powder diffraction files for: jacobsite (PDF\#OO-010-0319), bixbyite (PDF\#00010-0069), magnetite (PDF\#01-076-1849) and hematite (PDF\#00-024-0072).

de fragmentos de piezas cerámicas arqueológicas, ya que combinándolas e interpretando los resultados de manera conjunta, se logra una muy buena caracterización de los pigmentos empleados.

Hay que resaltar que con las tres técnicas se detecta presencia de manganeso en las muestras que contienen pigmentos negros, principalmente en la forma de jacobsita $\left(\mathrm{MnFe}_{2} \mathrm{O}_{4}\right)$ y también bixbyita $\left[(\mathrm{Mn}, \mathrm{Fe})_{2} \mathrm{O}_{3}\right]$, junto con óxido de hierro en la forma de magnetita $\left(\mathrm{Fe}_{3} \mathrm{O}_{4}\right)$. Considerando el bajo contenido de carbono observado, el pigmento utilizado en el color negro no puede ser atribuido a este elemento. Los rojos empleados mostraron ser fundamentalmente óxidos de hierro en forma de hematita $\left(\mathrm{Fe}_{2} \mathrm{O}_{3}\right)$.
La presencia de jacobsita en los pigmentos negros podría deberse a la aplicación directa del mineral sobre las piezas, previo a la cocción, ya que está presente en ambientes geológicos metamórficos (Katz 1960; Bowles et al. 2011). También podría surgir del uso de minerales ricos en Fe y Mn, que dieran lugar a la formación de jacobsita al ser sometidos a un tratamiento térmico o cocción con temperaturas mayores a $900^{\circ} \mathrm{C}$ (Schweizer \& Rinuy 1982). Considerando que los estudios petrográficos y experimentales efectuados previamente sobre pastas Vaquerías indican temperaturas de cocción de alrededor de $\operatorname{los} 800^{\circ} \mathrm{C}$ (Cremonte 1996; Pereyra Domingorena 2010), la presencia de jacobsita no podría explicarse por la cocción de las vasijas a altas temperaturas. 


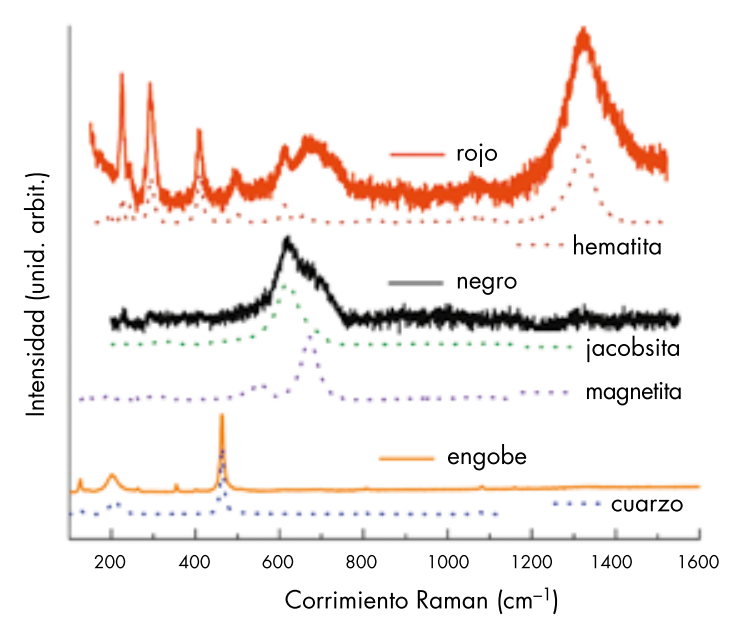

Figura 7. Espectros Raman de la muestra V3 en tres puntos diferentes: engobe, pigmento negro y pigmento rojo. Se incluyen en línea punteada espectros de referencia.

Figure 7. Raman spectra from sample V3 at three different points. slip, black pigment and red pigment. Library spectra are shown as dotted lines.
La aplicación de los diseños pintados de manera previa a la cocción de las cerámicas también ha sido observada mediante el análisis macroscópico estilístico de las terminaciones de las superficies pulidas. Varias piezas de este estilo presentan superficies manchadas por el quemado al fuego por encima de los diseños. Asimismo, se registra claramente el pulido en líneas efectuado antes de la cocción, cubriendo los motivos pintados.

Además, el estudio petrográfico de las pastas Vaquerías de Cardonal y Bordo Marcial demostró el uso de inclusiones no plásticas de tipo pizarra-filita. Las mismas son propias de ambientes metamórficos que no se corresponden con el ambiente geológico de la región donde se hallan estas aldeas, por lo que es probable que el lugar de producción de esta cerámica se encuentre ubicado en otras áreas vecinas (Pereyra Domingorena 2010; Bugliani \& Pereyra Domingorena 2012). Por esta razón, y a modo de hipótesis, se ha considerado a la

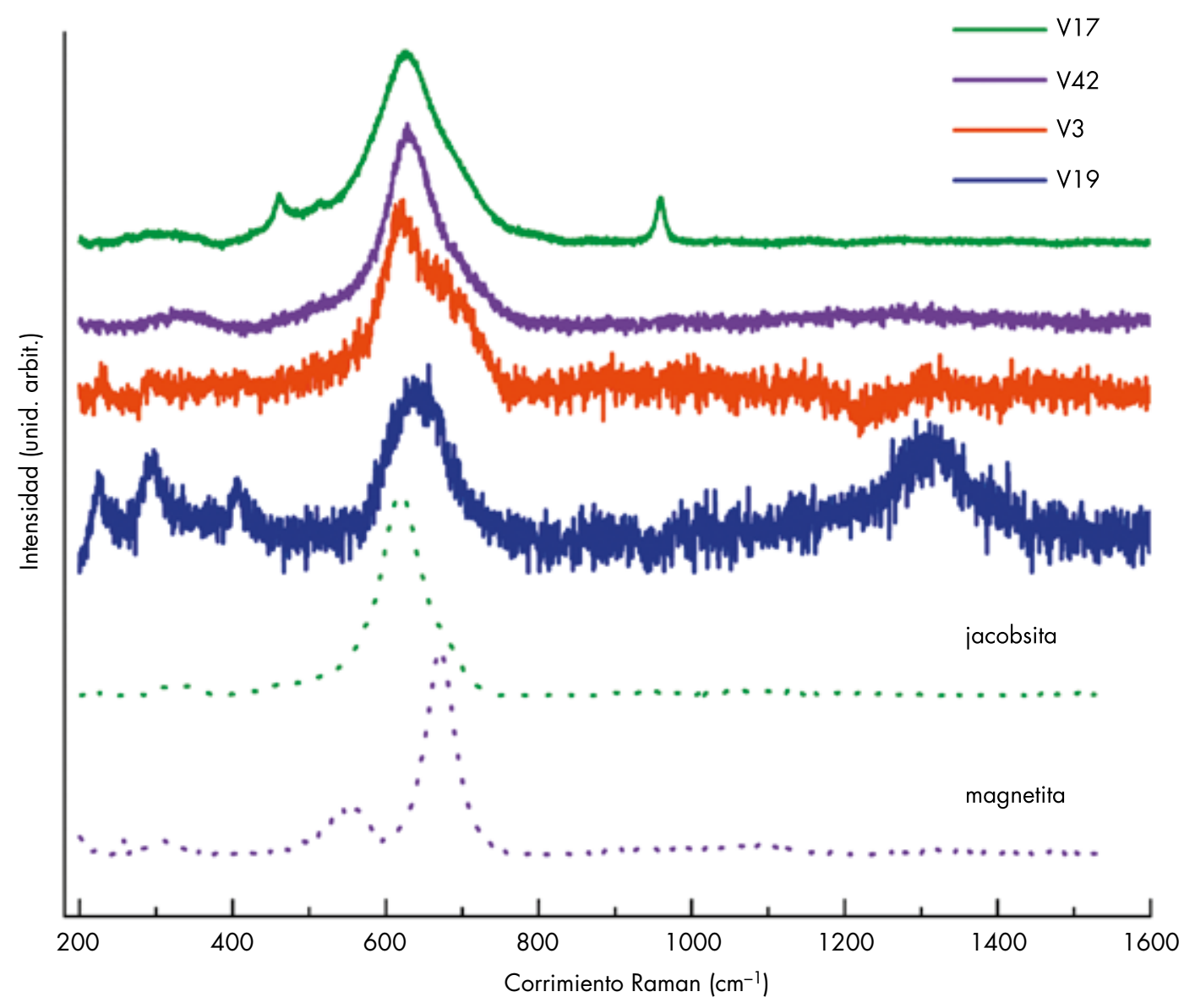

Figura 8. Espectros Raman de pigmentos negros en distintas muestras. Se incluyen en línea punteada espectros de referencia. Figure 8. Raman spectra from black pigment on several samples. Library spectra are shown as dotted lines. 
alfarería Vaquerías como material foráneo obtenido a través de las redes activas de circulación e interacción regional de bienes. La presencia de jacobsita en los diseños negros, un compuesto presente en ambientes geológicos metamórficos, sería congruente con los componentes observados en la composición de las pastas.

Esta contribución adiciona resultados significativos para avanzar en una definición más precisa del Estilo Vaquerías, el cual ha sido fundamentalmente caracterizado a partir del análisis de sus atributos plásticos y del diseño. Los resultados aquí obtenidos constituyen un importante avance para caracterizar los aspectos tecnológicos de este estilo cerámico pintado. Será preciso continuar estos estudios para determinar si es posible confirmar la presencia de jacobsita en la geología del NOA y profundizar en los procedimientos técnicos utilizados en la confección de estas vasijas polícromas.

\section{REFERENCIAS}

Botto I. L.; V. L. Barone, M. B. Cremonte \& M. A. SÁnchez, 1998. Estudios arqueométricos de cerámicas provenientes del Noroeste Argentino. Información Tecnológica 9 (6): 79-86.

Bowles J. F. W; R. A. Howie, D. J. Vaughan \& J. Zussman, 2011. Rock forming minerals: Non-silicates: Oxides, bydroxides and sulphides, Volume 5. Bath: Geological Society.

Bugliani M. F. \& L. Pereyra Domingorena, 2012. Una aproximación estilístico-tecnológica a la cerámica polícroma Vaquerías del Noroeste Argentino. Estudios Atacameños 43: 121-138.

Centeno, S. A.; V. I. Williams, N. C. Little \& R. J. Speakman, 2012. Characterization of surface decorations in pre-Hispanic archaeological ceramics by Raman spectroscopy, FTIR, XRD and XRF. Vibrational Spectroscopy 58: 119-124.

Cremonte, M. B., 1996. Investigaciones arqueológicas en la Quebrada de la Ciénega (Dpto. de Tafí, Tucumán). Tesis Doctoral. Facultad de Ciencias Naturales y Museo, Universidad Nacional de La Plata.
Cremonte, M. B.; M. Baldini \& I. L. Botto, 2003. Pastas y colores. Un camino al conocimiento del Estilo Portezuelo de Aguada. Intersecciones en Antropología 4: 3-16.

De Feo, M. E., 2010. Organización y uso del espacio durante el Período Formativo en la Quebrada del Toro (Pcia. de Salta). Tesis doctoral inédita. Facultad de Ciencias Naturales y Museo. Universidad Nacional de La Plata.

De la Fuente, G. A. \& J. M. Pérez Martínez, 2008. Estudiando pinturas en cerámicas arqueológicas Aguada Portezuelo ( $c a$. 600-900 AD) del Noroeste Argentino: Nuevos aportes a través de una aproximación arqueométrica por microespectroscopia de Ramán (MSR). Intersecciones en Antropología 9: 173-186.

González, A. R. \& M. BALDini, 1989. Vaquerías: La más antigua alfarería polícroma del Noroeste Argentino. Más allá del objeto 14: 8-12.

Heredia, O.; J. A. Pérez \& A. R. González, 1974. La antigüedad de la cerámica polícroma en el Noroeste Argentino. Revista del Instituto de Antropología V: 133-151.

Katz, G., 1960. Jacobsite from the Negev, Israel. American Mineralogist 45: 734-738.

Korstanje, M. A., 1995. Nuevas reflexiones en torno a Vaquerías, un estilo cerámico polémico. Cuadernos FHYCS-UNJU 5: 169-179.

LAZZARI, M., 2005. Travelling objects and spatial images: Exchange relationships and the production of social space. En Global Archaeological Theory. Contextual Voices and Contemporary Thoughts, P. P. A. Funari, A. Zarankin \& E. Stovel, Eds., pp. 191210. Nueva York: Kluwer.

Pereyra Domingorena, L., 2010. Manufacturas alfareras de las sociedades aldeanas del primer milenio DC al sur de los valles Calchaquíes. Tesis doctoral. Facultad de Filosofía y Letras, Universidad de Buenos Aires.

Scattolin, M. C.; M. F. Bugliani, L. I. Cortés, C. M. Calo, L. Pereyra Domingorena \& A. D. Izeta, 2009a. Pequeños mundos: Hábitat, maneras de hacer y afinidades en aldeas del valle del Cajón, Catamarca. Relaciones de la Sociedad Argentina de Antropología XXXIV: 251-274.

Scattolin, M. C.; L. Cortés, M. F. Bugliani, C. M. Calo, L. Pereyra Domingorena, A. D. IzeTA \& M. LAZZARI, 2009b. Built landscapes of everyday life: A house in an early agricultural village of northwestern Argentina. World Archaeology 41 (3): 396-414.

SChweizer, F. \& A. Rinuy, 1982. Manganese Black as an Etruscan Pigment. Studies in Conservation 27: 118-123.

Tartusi, M. R. A. \& V. A. Núñez Regueiro, 1993. Los Centros Ceremoniales del NOA. Publicaciones del Instituto de Arqueología 5 (Serie: Ensayos 1): 1-49. 